\title{
Accelerated flow of an incompressible fluid with wake formation.
}

Memoria di Thúodore oe Kármán (a Pasadena).

\begin{abstract}
Summary. - The methods of conformal transformation are applied to the nonsteady flow of an incompressible fluid around a flat plate with wake formation. The condition for a closed aren of dead fuid following the plate is established.
\end{abstract}

The theory of the stationary flow of an incompressible fluid with wake formation has been treated in great details in the scientific literature. Especially the great Italian master of mathematical hydrodynamiis, LEvi-CIVITA, gave quite a general method to compute two dimensional flows having partly free boundaries. This short publication essentially follows Levi-Crvita's method, but application is made to accelerated flow. The main interest of the calculation is that one finds a flow pattern round a flat plate moving with certain given acceleration thru the fluid in rest, so that a closed dead water region follows the plate. This flow pattern can be fully described by elementary functions.

Instead of the accelerated motion of the plate, we consider an accelerated motion of the fluid which is extended to infinity around a plate at rest. The velocity of the flow at infinity be denoted by $U(t)$, the acceleration $\frac{d U}{d t}$ by $a$. We want to investigate the conditions for a flow pattern which remains similar as function of the time $t$, the velocity at every point being multiplied by a factor which is a function of the time only. Then the complex potential function $F(z)$ has the form

$$
F(z)=U f(z)
$$

where $z=x+i y$ and $x$ and $y$ are Cartesian coordinates in the plane in which a two dimensional flow takes place.

We consider the flow produced by the presence of a plate of the width $2 h$, placed in the stream perpendicular to the flow direction. The point $A$ represents infinity, $B$ the stagnation point, $C$ the point of separation of the flow and - as indicated above - we will assume that the two branches of the flow close again at a point $D$.

If the fluid in the wake is at rest, the pressure must be constant along 
the free boundary $C D$. The condition of constant pressure is

$$
\frac{\partial \Phi}{\partial t}+\frac{1}{2}|w|^{\circ}=\text { const. }
$$

where $\Phi$ is the real part of $F$ and $w$ is the absolute value of $w=u-v i$, $u$ and $v$ being the components of the velocity in the $x$ and $y$ directions respectively. From (1) it follows that Eq (2) can be written in the form

$$
\alpha \varphi+\frac{1}{2}\left[\left(\frac{\partial \varphi}{\partial x}\right)^{2}+\left(\frac{\partial \varphi}{\partial y}\right)^{2}\right]=\text { const. }
$$

where $\varphi$ is the real part of $f$.

The problem is therefore to determine a functional relation between $f$ and $w$ such that $w$ is an analytical function of $f$ and

1) between $A$ and $B$ and between $D$ and $A, v=0$

2) between $B$ and $C, u=0$

3) between $C$ and $D$, the equation (3) is satisfied

4) at infinity, $w=U$.

We ehoose an intermediary variable $\zeta$, such that

$$
f=-A h\left[1+\frac{1}{2}\left(\zeta+\frac{1}{\zeta}\right)\right]
$$

It is seen that $\mathrm{Eq}(4)$ represents a component transformation of the half of the $f$ plane into the interior of a circle in the $\zeta$ plane. $A$ is a non-dimensional constant.

We want to specify the transformation between the $f$ and $\zeta$ planes in such a way that the point $A$ shall correspond to $\zeta=0$, the point $B$ to $\zeta=k$ where $k$ is a positive real quantity smaller than unity, the point $C$ to $\zeta=1$, and the point $D$ to $\zeta=-1$. It is seen that the circle $\zeta=e^{1 \alpha}$ corresponds to the free boundary.

It is easy to see that the function

$$
w=U(1+\zeta) \sqrt{\frac{1-\zeta / k}{1-k \zeta}}
$$

satisfies the conditions 1), 2) and 4). As to the condition 3) - relating to the free boundary - Eq (3) becomes

$$
-A h a(1+\cos \alpha)+\frac{U^{2}}{k}(1+\cos \alpha)=\text { const. . }
$$

The left side is evidently equal to zero and therefore does not vary along the semi-circle, if the constant $A$ is chosen to be equal to

$$
A=\frac{U^{2}}{k h a} \text {. }
$$


One more condition is, however, to be satisfied if Eqs (4) and (5) shall truly represent the flow pattern which we want to establish: the point $D$ in the $z$ plane has to lie on the real axis $x$. Therefore the imaginary part of the integral $\int_{B}^{D} d z=\int_{B}^{D} \frac{d F}{w}$ must vanish. It is not difficult to show that this condition is satisfied if the residuum of the integral $\int \frac{d f}{w}$ taken around the point $\zeta=0$ finishes. This furnishes the condition $\frac{1}{k}-k=2$ or $k=\sqrt{2}-1=.414$.

The numerical constant $A$ is determined by the condition that the distance between the points $B$ and $C$ in the $z$ plane must be equal to $h$ i.e. to the half width of the plate. Consequently

$$
\int_{B}^{C} \frac{d F}{w}=-\frac{A h}{2} \int_{i c}^{1} d \zeta\left(1-\frac{1}{\zeta^{2}}\right) \frac{1}{1+\zeta} \sqrt{\frac{1-\hbar \zeta}{1-\zeta_{k}}}=-h i .
$$

The integral $B$ c $: n$ be easily evaluated and we obtain (with $k=\sqrt{2}-1$ )

$$
\frac{1}{A}={ }_{2}^{1}\left[\sqrt{\sqrt{2}-1}+(\sqrt{2 \cdot}-1) \cos ^{-1}(\sqrt{2}-1)\right]=.692 .
$$

The physical meaning of this relation is the following: the non dimensional quantity $\frac{U^{2}}{a h}$ can be considered as the characteristic « Froude number" of the accelerated motion. Evidently a closed wake of constant shape can Le obtained only for one value of this Froude number, namely

$$
\frac{U^{2}}{a h}=k A=.596 \text {. }
$$

The corresponding velocity versus time relation can be witten in the form

$$
U=\frac{U_{0}}{1-1.675 \frac{U_{0} t}{h}}
$$

where $U_{0}$ is the velocity of the flow at $t=0$.

The question arises what will be the flow pattern for other values of the Froude number? Probably the closed wake formation which we found above is the only pattern in which the shape of the wake is invariant with time. Therefore in the general case the mathematical problem is much more com. plicated but certainly can be solved. I should be gratified if some younger scholar of mathematical hydrodiynamics would complete these investigations for the general case. 